\title{
PERFIL SOCIODEMOGRÁFICO Y CARACTERIZACIÓN DE LAS EMPRESAS DE LA MUJER EMPRENDEDORA EN ESPAÑA. FORTALEZAS Y DEBILIDADES
}

\author{
Ángel José Olaz Capitán \\ Pilar Ortiz García \\ Departamento de Sociología y Trabajo Social. Facultad de Economía y Empresa \\ Universidad de Murcia
}

DOI: $10.1387 /$ lan-harremanak.15413

\section{ABSTRACT}

En las últimas décadas la investigación sobre el emprendimiento está adquiriendo un importante protagonismo como resultado de los cambios experimentados en esta forma de actividad empresarial. La presencia de la mujer en este ámbito de actuación es cada vez más evidente como reacción ante las mayores dificultades de acceso al empleo en un contexto de crisis y no tanto a la visión romántica de una «idea luminosa» que se materializa por generación espontánea. En este contexto el impulso a la iniciativa emprendedora que las mujeres están desarrollando adquiere una especial singularidad.

Tomando como principal fuente de referencia la explotación de los datos del proyecto GEM 2012 —última actualización disponible—, esta comunicación profundizará en el estudio del perfil sociodemográfico de la mujer emprendedora en España en el intento por caracterizar las singularidades de las empresas en las que éstas desarrollan su actividad. 
PERFIL SOCIODEMOGRÁfico Y CARACTERIZACIÓN DE LAS EMPRESAS DE LA MUJER EMPRENDEdORA EN ESPAÑA.

La comparativa por géneros presenta importantes elementos de análisis atendiendo a variables tales como: expectativas de crecimiento del empleo, razones de abandono de la empresa, motivaciones de emprendimiento por necesidad, orientación internacional de la empresa y otros tantos elementos que se estudiarán.

A través de estas primeras conclusiones, el proposito de esta comunicación es reflexionar sobre las características que hacen de la mujer una potencial emprendedora y, por consiguiente, anticipar medidas que contribuyan a su mejor visibilización y puesta en valor como elemento clave en el desarrollo laboral, social y económico de nuestra sociedad.

Palabras clave: Género, mujer, emprendimiento, competencias, empleo.

Recently, research on entrepreneurship is acquiring an important role as a result of the changes experienced in this form of business. The presence of women in this scope is increasingly apparent, reaction to the greater difficulties of access to employment in a context of crisis rather than to the romantic vision of a "brilliant idea" that materializes out of nowhere.

Taking as reference the source of data exploitation GEM 2012, this communication deepen the study of the demographic profile of women entrepreneurs in Spain in an attempt to characterize the singularities of the companies in which they work.

The gender analysis has important elements of response to such variables as employment growth expectations, reasons for leaving the company, motivation of necessity entrepreneurship, international orientation of the enterprise and many other elements to be studied.

Through these initial findings, the purpose of this communication is to reflect on the characteristics that make a potential entrepreneurial women and therefore anticipate measures that contribute to their better visibility and value as a key element in career development, social and economic development of our society.

Key Words: Gender, woman, entrepreneurship, skills, employment. 


\section{Introducción}

La investigación ${ }^{1}$ sobre el emprendimiento está adquiriendo un importante protagonismo como resultado de los cambios experimentados en esta forma de actividad empresarial en los últimos años y muy posiblemente debido al impacto de la crisis económica.

Asimismo, la atención prestada a esta cuestión en ámbitos económicos y académicos viene determinada por la confianza puesta en la actividad emprendedora, como generadora de innovación, creatividad y crecimiento.

La presencia de la mujer en este ámbito de actuación no puede pasar inadvertida. Dicha relevancia y evolución es interpretada como una reacción de éstas ante las mayores dificultades de acceso al empleo en un contexto de crisis. Ante esta situación, el impulso a la iniciativa emprendedora en las mujeres adquiere una especial singularidad. Constituye pues, un reto de esta sociedad conseguir unas mayores cotas de empleabilidad de la mujer, ya que esto constituye una premisa para alcanzar una mayor igualdad.

Es en este contexto donde se gesta el proyecto Mujer y Emprendimiento desde una Perspectiva Competencial (CSO2013-43667-R) financiado por el Ministerio de Economía y Competitividad y que da lugar al equipo de investigación formado por una quincena de profesores investigadores de la Universidad de Murcia y la Bradford (Reino Unido).

De las distintas variables que han sido consideradas para determinar los factores que promueven el emprendimiento femenino, la perspectiva competencial no ha sido una vía suficientemente explorada.

\footnotetext{
${ }^{1}$ Esta comunicación presentada por los investigadores principales del Proyecto: «Mujer y Emprendimiento desde una Perspectiva Competencial» (CSO2013-43667-R), financiado por el Ministerio de Economía y Competitividad, es una síntesis del Documento Interno de Trabajo «Definición del universo poblacional de empresas dirigidas por mujeres emprendedoras y su perfil», realizado junto al equipo de investigación de la Universidad de Murcia formado en esta fase del proyecto por: Juan José García Escribano, Maribel Sánchez-Mora Molina, María Belén García Palma, Nuría Nevers Esteban Lloret y Alicia Rubio Bañón.
} 
Bajo este marco y con el propósito de analizar el perfil competencial de la mujer emprendedora, el equipo de investigación se encuentra en la fase de detección de los puntos fuertes y débiles del emprendimiento femenino, poniendo especial atención en los aspectos relativos a la formación, capacidad y habilidades de ésta. Es, a través de este diagnóstico, donde se contribuirá a reforzar la capacidad competitiva de las mujeres emprendedoras junto al diseńo de acciones específicas que favorezcan el desarrollo de un plan de empresa o consolidar y modernizar el ya existente.

Estos aspectos suscitan la reflexión acerca de que el emprendimiento entra en la lógica de la regeneración permanente del sistema empresarial, sin el cual, el modelo productivo actual difícilmente podría sobrevivir. Bajo esta filosofía, el Proyecto Global Entrepreneurship Monitor $(\mathrm{GEM})^{2}$, a partir del examen de la actividad emprendedora de más de 70 países, pone de manifiesto que la ratio entre el porcentaje de mujeres y hombres emprendedores es desfavorable para la mujer.

Tomando como principal fuente de referencia la explotación de los datos del proyecto GEM, la presente comunicación está estructurado del siguiente modo. En primer lugar, se comenzará por estudiar el perfil sociodemográfico de la mujer emprendedora en España como preámbulo a un intento por caracterizar las singularidades de las empresas en las que ellas desarrollan su actividad. Este primer análisis quedaría, sin embargo, no suficientemente perfilado sino se relaciona esta panorámica nacional con su correlato regional, tanto en el perfil sociodemográfico como en su caracterización empresarial. Tras esta objetivación de los diferentes ámbitos empleados se procedera a desarrollar las oportunas conclusiones.

De este modo, creemos haber cubierto un primer objetivo inicial en cuanto a definición del perfil emprendedor de la mujer, todo ello sin menoscabo de ir completándolo más adelante en los sucesivas etapas de este proyecto de investigación.

2 El Proyecto GEM y más concretamente su observatorio del emprendimiento fue ideado por los profesores Michael Hay y Bill Bygrave de la London Business School y del Babson College, respectivamente, en el ańo 1997 y desarrollado en su parte técnica por el profesor Paul Reynolds en sus primeros siete años de vida. Es en año 1999 cuando se llevó a cabo una edición piloto con la participación de los países del Grupo de los 7, más Dinamarca, Finlandia e Israel, abriéndose el proyecto a todo el mundo en el 2000 , momento en el que nuestro país se asoció al estudio a través de la Escuela de Negocios Instituto de Empresa Business School. En el momento actual, la Red GEM Espańa está constituido por una Red de Equipos implantados en la mayoría de Comunidades y Ciudades Autónomas, de la que forma parte la Universidad de Murcia a través de la Cátedra de Emprendedores.

Tras dieciséis años de andadura, el proyecto se ha consolidado como la fuente de información más completa sobre emprendimiento a nivel internacional, con una participación regular de más de cincuenta países. Se han creado más de setenta equipos GEM en otras tantas naciones y, Espańa, lidera el observatorio junto con el Reino Unido, aportando bases de datos que cuentan con muestras de más de 28.000 entrevistas a la población de 18 a 64 años de edad. 


\section{Perfil sociodemográfico y caracterización de las empresas de la mujer emprendedora en España}

\subsection{Perfil sociodemográfico de las mujeres emprendedoras en España}

La mujer juega un importante papel en la actividad emprendedora en Espańa, por ello, cada vez son más los estudios centrados en identificar el perfil de estas empresarias, así como las características de los negocios que emprenden.

Una de las fuentes más completas para conocer ambos aspectos es el Proyecto Global Entreprenerusihip Monitor (GEM). Los informes generados por este proyecto sirven de base para trazar el perfil sociodemográfico de los emprendedores en función de género y las características que a continuación se sintetizan.

Según los datos de GEM para 2012 para España no existen grandes diferencias en el perfil de hombres y mujeres emprendedores. En concreto, presentan similares características tanto por edad, como por núcleo familiar de pertenencia. En ambos casos la edad media está en torno a los 38 años y tanto hombres como mujeres son miembros de núcleos familiares compuestos por un número ligeramente superior a los tres miembros.

No obstante, sí se aprecia alguna diferencia en otros aspectos tales como el nivel de estudios y de renta, así como en el origen geográfico. En cuanto al primero de los aspectos, la formación, el Informen GEM seńala que las mujeres suelen tener una formación ligeramente superior a la de los emprendedores varones. En concreto, en 2012 el porcentaje de emprendedoras con un nivel de estudios universitario es de 38,4\% frente al 32,3\% de este mismo nivel en el caso de los hombres.

También hay que constatar la diferencia entre ambos sexos en cuanto al nivel de renta, en este caso, a favor de los emprendedores varones, un hecho que el Informe GEM atribuye al tipo de empresa que crean unos y otras y que, en el caso de las mujeres, tiene un carácter más convencional, menos tecnológico y más orientada al consumo.

Aunque el emprendimiento en España es fundamentalmente de nativos, hay que señalar una última singularidad respecto al origen geográfico de los emprendedores. Según los datos disponibles en el informe a que se viene haciendo referencia, las mujeres extranjeras son ligeramente más emprendedoras que los varones en España.

\subsection{Caracterización de las empresas de las mujeres emprendedoras en España}

En lo referente a la caracterización de las empresas de mujeres emprendedoras en nuestro país, una de las características más reveladora es la referente al 
tamaño. La dimensión de las empresas es muy reducida, ya estén creadas por hombres o por mujeres. Como ya pone de manifiesto el Informe Global Entrepreneurship Monitor (GEM) de 2012, más del 90\% — en ambos casos- son empresas de menos de cinco trabajadores. Se observa, no obstante, que el porcentaje de empresas lideradas por mujeres entre 1 y 5 trabajadores $(36,8 \%)$ es ligeramente superior al de las lideradas por hombres $(34,9 \%)$, mientras que en el tramo inmediatamente superior — de 6 a 19 trabajadores - es algo superior el porcentaje de empresas emprendidas por hombres $(6,6 \%)$ que el de empresas emprendidas por mujeres $(5,8 \%)$. Se trata, en cualquier caso, de micro-pymes si nos atenemos al dato de que el $57,2 \%$ de empresas creadas por hombres y el $56,6 \%$ de las empresas constituidas por mujeres, no cuenta con «ningún trabajador» (gráfico 1).

\section{Gráfico 1}

Número de trabajadores y sexo del emprendedor.

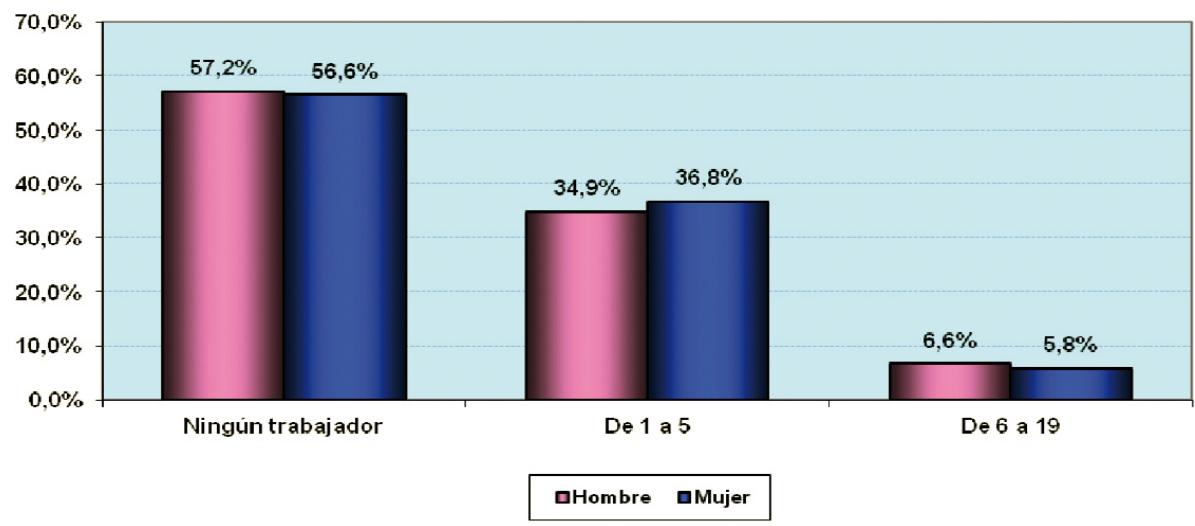

Fuente: Elaboración propia.

Globalmente, y atendiendo a la evolución respecto al año anterior facilitada por el GEM, el tamaño ha subido ligeramente, dado que asciende el porcentaje de empresas de 1 a 5 y 6 a 19 trabajadores y desciende el de las que no cuentan con ningún trabajador.

Estos datos son coherentes con los facilitados por el GEM sobre el número de propietarios (gráfico 2), dado que más del $50 \%$ de empresas tiene un único propietario, tanto entre las iniciativas femeninas como entre las masculinas, aunque parece existir una mayor proporción de iniciativas de mujeres promovidas por 2, 3 o 4 propietarias, que entre las iniciativas protagonizadas por hombres. La tendencia se invierte cuando el número de propietarios es igual o superior 
a 5 ya que, en este caso, el porcentaje de empresas emprendidas por hombres $(4,6 \%)$ es superior al de creadas por mujeres $(1,9 \%)$. No obstante, de estos datos se podría inducir una ligera mayor propensión al emprendimiento cooperativo en el caso de las mujeres, al menos cuando se trata de un número reducido de propietarias.

\section{Gráfico 2}

Número de propietarios en función del sexo

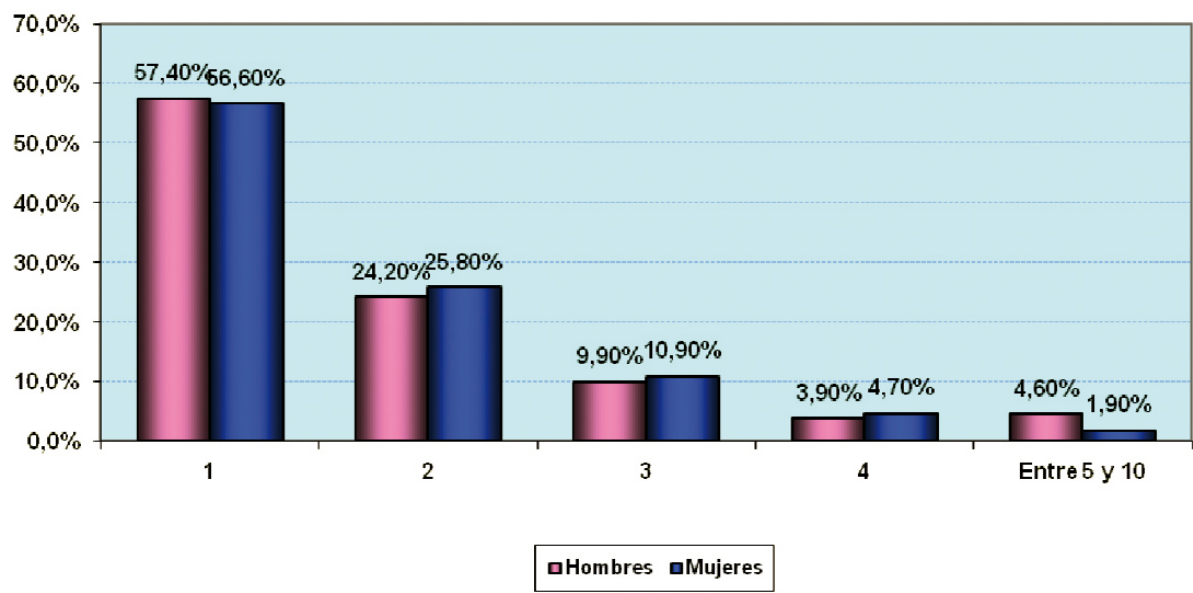

Fuente: Elaboración propia.

Gráfico 3

Expectativas de crecimiento del empleo en función del sexo

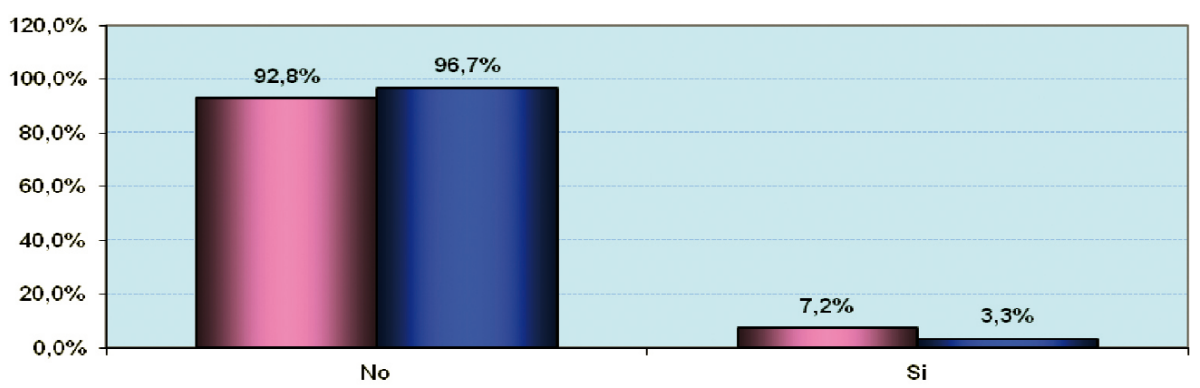

DHombres aMujeres

Fuente: Elaboración propia. 
En general las expectativas de crecimiento de la empresa no son buenas, independientemente del sexo, aunque tal como se aprecia en el gráfico 3 existe un mayor pesimismo entre las iniciativas femeninas, dado que el $96,7 \%$ considera que no crecerá, frente al $92,8 \%$ de las empresas lideradas por hombres que considera esta opción.

Sin duda, el largo período de crisis que viene soportando la economía española es un factor modulador de las expectativas empresariales en general y, en particular, entre quienes tradicionalmente afrontan mayores dificultades en el ámbito laboral, como son las mujeres.

En consonancia con la valoración sobre la perspectiva general de crecimiento de la empresa, la opinión sobre las posibilidades de generación de empleo es también cautelosa entre los emprendedores. Según se aprecia en el gráfico 4 sólo el 8,3\% de mujeres emprendedoras prevé el crecimiento en más de cinco empleados en los próximos cinco años; un porcentaje que asciende al $15,1 \%$ cuando los que responden a la misma cuestión son los hombres. Parece, por tanto, que las mujeres son menos optimistas en lo que se refiere al crecimiento general de la empresa y del empleo en particular. Por otra parte, la percepción sobre las posibilidades de expansión de la empresa ha sido decreciente según el informe GEM sobre esta misma cuestión en 2011.

\section{Gráfico 4}

Expectativas de creación de más de cinco empleos en los próximos cinco años

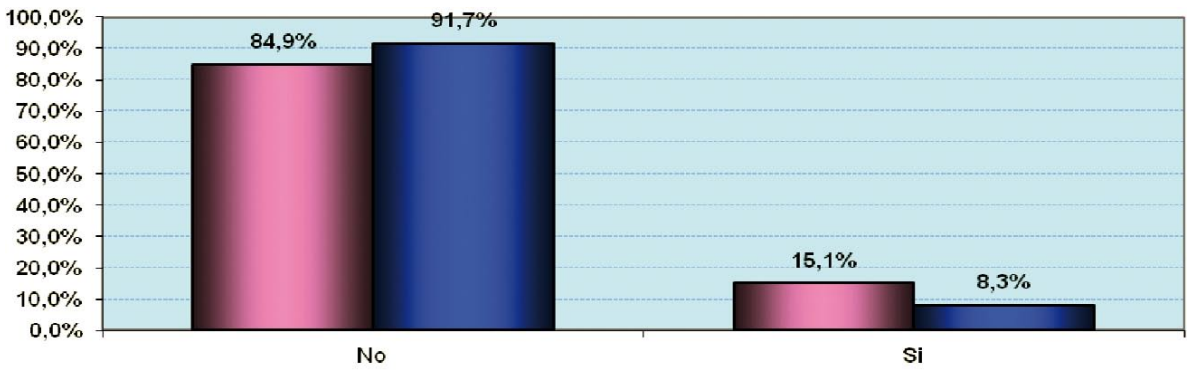

口Hombres aMujeres

Fuente: Elaboración propia.

El grado de intensidad exportadora de las empresas es bajo a pesar de que los datos GEM de 2012 ponen de manifiesto un incremento respecto al año anterior. No existen notables diferencias en razón de género, aunque es reseñable el hecho de que las empresas de iniciativa femenina exportan en menor me- 


\section{Gráfico 5}

Grado de exportación de las empresas exportadoras en función del sexo

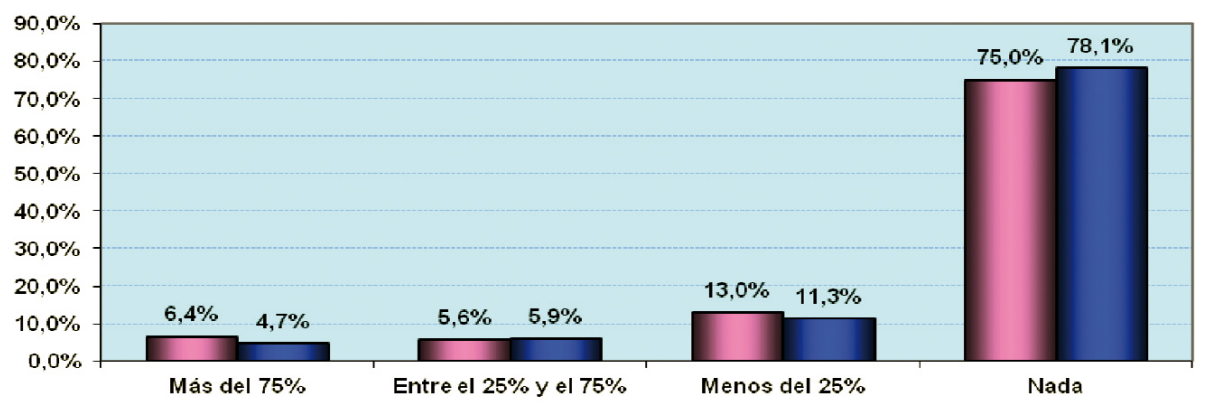

口Hombres aMujeres

Fuente: Elaboración propia.

dida que aquellas emprendidas por hombres. Concretamente, como se aprecia en el gráfico 5 , un $78,1 \%$ de las primeras dicen no exportar «Nada», un porcentaje que desciende al $75 \%$ en el caso de las empresas cuyo emprendedor es un hombre.

Según el informe del GEM de 2012 las tasas de abandono de hombre y mujeres son similares, no obstante, existen algunas diferencias de interés en cuanto a los motivos por los que se porduce el abandono de la empresa (gráfico 6). De

\section{Gráfico 6}

Razones de abandono de la empresa en función del sexo

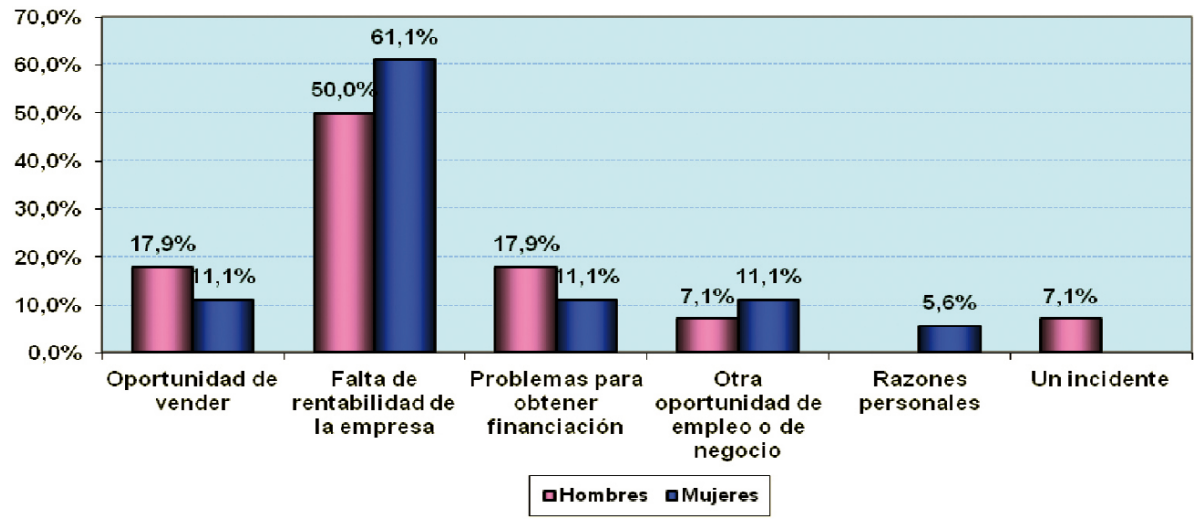

Fuente: Elaboración propia. 
estas diferencias una de las más significativas es la que se produce en torno a la falta de rentabilidad, uno de los motivos principales por los que se opta por el cierre y que en el caso de las iniciativas femeninas supone el 61,1\% de dichos motivos, frente al $50 \%$ en el caso de las empresas creadas por sus homólogos varones.

Otras razones de cierre como los "problemas para obtener financiación» o la «oportunidad de vender» también presentan diferencias en razón de sexo del propietario y en ambos casos es superior el porcentaje de emprendedores varones que abandonaría el negocio por estas causas (17,9\% en ambos casos) que el de mujeres ( $11,1 \%$ en ambos casos también). En cuanto al cierre ante «otra oportunidad de empleo o negocio", aunque es minoritario entre los empresarios, es superior el porcentaje de mujeres para las que esta razón sería motivo de cierre $(11,1 \%)$ que el de hombres $(7,1 \%)$. Así ocurre también cuando lo que se aduce son «razones personales».

Continuando con las razones, pero en este caso, para emprender, la disyuntiva entre oportunidad y necesidad es una de las cuestiones básicas. El informe GEM pone de manifiesto que el emprendimiento en España responde, en líneas generales, al reconocimiento de una oportunidad. Sin embargo, la actividad emprendedora de la mujer aparece ligada de forma significativa a la «necesidad». Los datos del gráfico 7 evidencian esta diferencia en razón de sexo ya que el $28,7 \%$ de iniciativas femeninas en la fase inicial de la actividad, dicen haberse producido por este motivo. En el caso de las iniciativas masculinas, este porcentaje desciende al $24,7 \%$.

\section{Gráfico 7}

Razones de emprendimiento por necesidad en función del sexo*

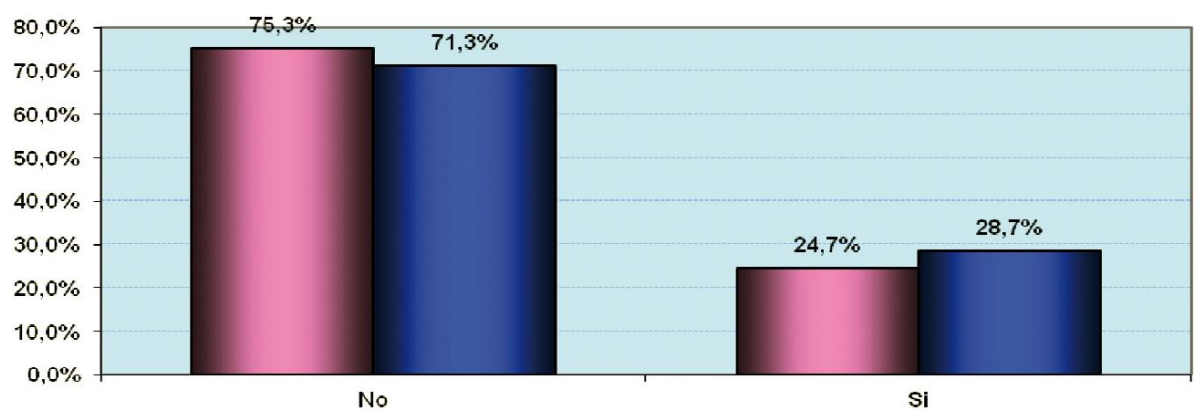

aHombres aMujeres

* Sobre la muestra de emprendedores en la fase inicial.

Fuente: Elaboración propia. 
No existe diferencia significativa por sexo en cuanto al grado de novedad que aportan las iniciativas emprendedoras (gráfico 8), si bien, el grado de novedad total o parcial del producto que se oferta es algo inferior en el caso de las empresas de iniciativa femenina $(37,7 \%$ en este caso y $38,9 \%$ en las constituidas por hombres).

\section{Gráfico 8}

Grado de novedad del producto para la totalidad o mayoría de consumidores en función del sexo*

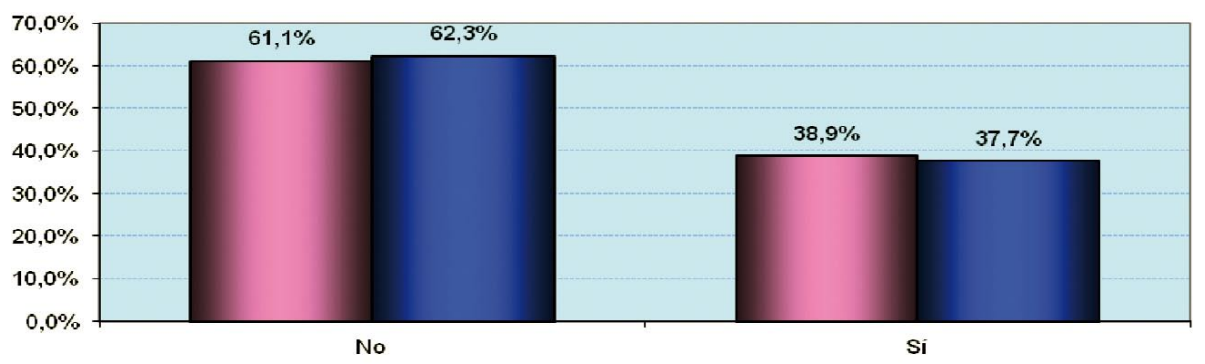

aHombres aMujeres

Fuente: Elaboración propia.

El informe del GEM detecta en esta cuestión un ligero incremento en iniciativas novedosas respecto a 2011 independientemente del sexo del emprendedor.

Si bien el grado de novedad en de los productos de estas empresas no es alto, como se ha visto en el gráfico anterior, lo es en mayor medida respecto a las empresas competidoras. Tampoco en este caso es reseñable la diferencia en función del sexo aunque, como en el caso de la novedad del producto para el consumidor, también en este caso la novedad del producto ofertado por las mujeres emprendedoras respecto a sus competidoras es ligeramente inferior $(50,2 \%)$ al de las iniciativas masculinas $(50,9 \%)$ (gráfico 9).

En cuanto a la internacionalización de la empresa, es destacable que en general las iniciativas emprendedoras presentan un bajo grado de proyección internacional. En este caso, sólo el 10,8\% de las empresas de mujeres presenta este carácter. En el caso de los varones, este porcentaje sube ligeramente hasta el $12 \%$ (gráfico 10). 


\section{Gráfico 9}

Grado de novedad del producto entre empresas (pocas o ninguna empresa ofrece el producto) en función del sexo*

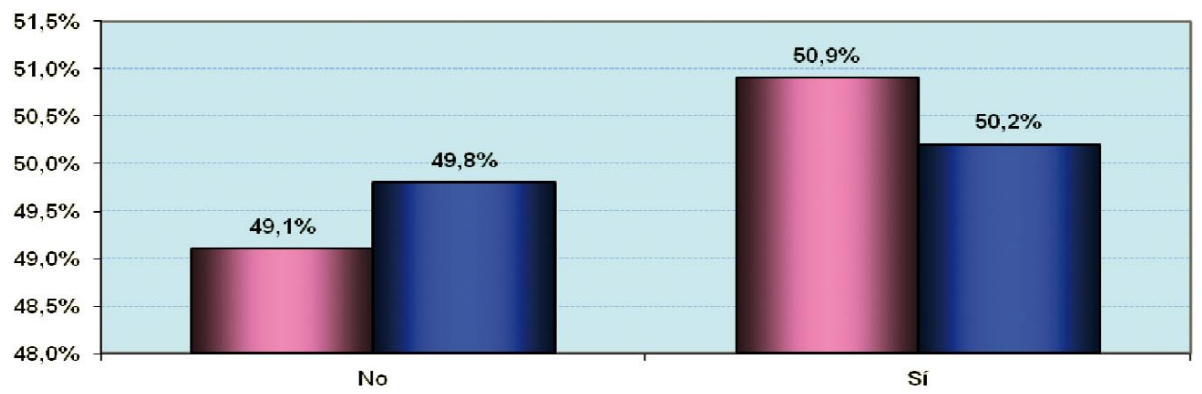

DHombres DMujeres

Fuente: Elaboración propia.

Gráfico 10

Fuerte orientación internacional de la empresa en función del sexo*

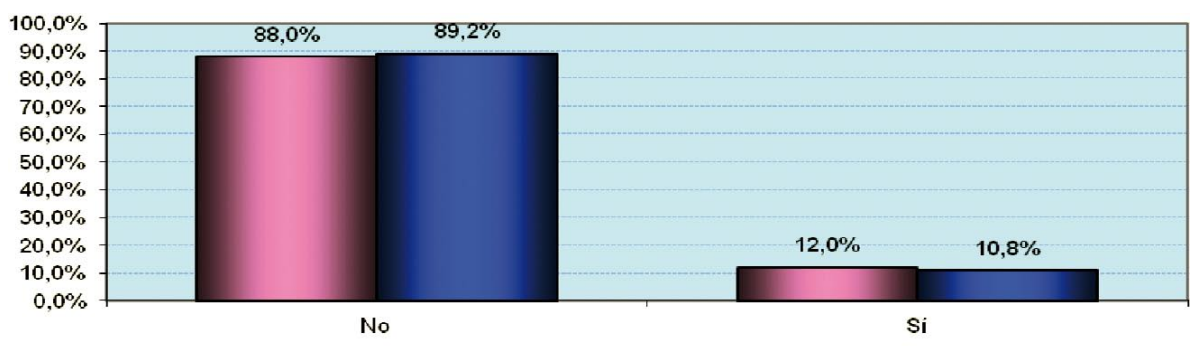

Fuente: Elaboración propia.

En clara lógica con esta constatación, la percepción sobre una débil orientación hacia la internacionalización se hace patente en las respuestas del empresariado (gráfico 11). En concreto, un 77,9\% de emprendedoras mujeres reconocen la debilidad de su proyección internacional y, con una escasa diferencia, aunque algo inferior, se manifiestan en el mismo sentido los emprendedores varones (75\%).

En suma, aunque no se establecen grandes diferencias en el emprendimiento en razón de género en las cuestiones analizadas, aparecen algunas peculiaridades 


\section{Gráfico 11}

Débil orientación internacional de la empresa en función del sexo*

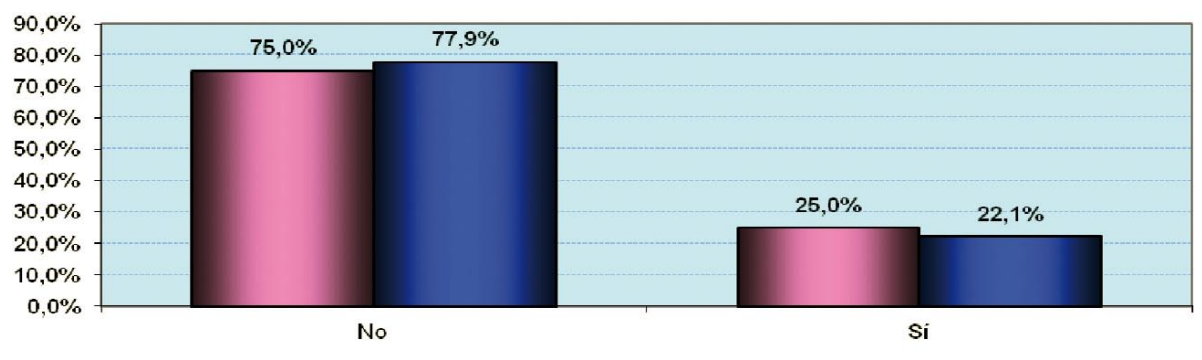

Fuente: Elaboración propia.

entre las que destacar el menor optimismo de la mujer respecto a las expectativas de crecimiento de la empresa (en negocio o empleo); en segundo lugar, también hay diferencia en cuanto a las causas por las que unas y otros optarían por el cierre de la empresa, en concreto, los motivos personales que para la mujer podrían ser una causa (aunque muy minoritaria), no aparecen mencionados entre los emprendedores varones, como tampoco el abandono de la empresa anter otra oportunidad de negocio o empleo. Se trata de cuestiones que podrían tener un significado importante si se interpretan a la luz de la teoría de género. En tercer lugar, ligado a las diferencias en la motivación para emprender, las iniciativas de las mujeres aparecen más claramente ligadas al factor necesidad que las de los hombres.

Por último, desde un punto de vista estadístico, hay que señalar que las diferencias en el emprendimiento en función de género son significativas únicamente en cuestiones relacionadas con las expectativas de crecimiento general y en el empleo en particular en las dimensiones analizadas.

\section{Conclusiones}

No siempre es tarea sencilla sintetizar de un modo preciso los principales aspectos de un estudio, más si cabe si este se encuentra en sus primeros compases. No obstante, el análisis realizado en lo referente tanto a su perfil sociodemográfico como a la caracterización de las empresas en las que éstas desarrollan su actividad, apuntan a interesantes reflexiones que se desarrollarán a continuación.

En lo referente al perfil sociodemográfico — según los datos de GEM (2012)— no existen a nivel nacional importantes diferencias en el perfil de las mujeres y 
hombres emprendedores, al contrario, se evidencian ciertas similitudes por razón de edad (entorno a los 38 años) y núcleo familiar de pertenencia (3 miembros).

No obstante, si se observa alguna ligera matización en lo concerniente al nivel de estudios y de renta, así como en el origen geográfico de sus protagonistas. En cuanto al primero de los aspectos, la formación universitaria es mayor en las féminas (38,4\% de las que en el caso de los hombres 32,3\%). En lo que se refiere a la renta - más elevada en el género masculino- permite emprender proyectos de naturaleza tecnológica, no así en el caso de la mujer que, con menos recursos, se centra en proyectos relacionados con el consumo. Por último y en lo referente a la procedencia geográfica de las emprendedoras parece observarse un mayor nivel emprendimiento por parte de las mujeres de origen extranjero que en el caso de las autóctonas.

En lo referente a cuestionas más directamente relacionadas con la caracterización de las empresas, según el observatorio GEM España 2012, éste señala que la distribución del género en las diferentes fases del proceso emprendedor es menor en las mujeres (40\%) que en los hombres (60\%).

En el caso del colectivo femenino, el emprendimiento en las denominadas fases activas (naciente, nueva, emprendedora totas y consolidada) queda incluso por debajo del $40 \%$. En cuanto a la evolución de los indicadores de actividad, en el comportamiento de la fase potencial puede observarse cierta estabilidad, mientras que disminuye el porcentaje de mujeres en la fase naciente, nueva, emprendedora y en los abandonos.

Todas estas cuestiones invitan a pensar que la actividad emprendedora de la mujer pone de manifiesto dos aspectos esenciales. Por una parte, la menor propensión a emprendimiento femenino con relación a los hombres y, por otra, que se trata de una actividad que va creciendo en los últimos ańos, tanto en el entorno internacional como en España. Por estas y por otras tantas razones, la relevancia del emprendimiento femenino y sus características distintivas respecto al de los hombres, sugieren la oportunidad de seguir abordando un análisis diferenciado.

Desde esta óptica cualitativa, parece desprenderse que los hombres muestran mayor optimismo que las mujeres en cuanto a la expansión de su empresa y la generación de empleo nuevo, así como en la innovación en el producto. Este optimismo bien pudiera estar fundamentado en la construcción social de una realidad en la que es el hombre es quien asume la labor productiva y la mejor reproductiva en el ámbito doméstico.

Así pues y concluyendo en este ejercicio de síntesis, se hace necesario incidir en la necesidad urgente de definir políticas, programas y planes de actuación específicos que impulsen el emprendimiento femenino, así como la mejora en la calidad de las iniciativas de negocio planteadas, profundizando en la especificidad y rasgos cualitativos de la actividad emprendedora. 
Aspectos de distinta naturaleza tales como el redimensionamiento de la ubicación geográfica, las facilidades hacia la financiación y un conjunto de acciones tomando como eje el aprendizaje y la adquisición, estimulación y desarrollo de los conocimientos, capacidades y habilidades (personales, sociales y profesionales), junto a una acción tutelada por parte de la Administración Nacional, Regional, asociaciones y el mundo académico se hacen clave en el desarrollo de este proceso.

En este sentido, el análisis del emprendimiento femenino a partir de las competencias que se despliegan y acompañan a una iniciativa de este carácter, es punto de partida para comprender los aspectos motivacionales de la actividad y, sobre todo, contribuir al desarrollo de acciones y el fomento de la calidad en este apasionante reto de nuestra sociedad actual.

\section{Bibliografía}

Informe Global Entrepreneurship Monitor (2012). España 2012, Madrid, Ministerio de Industria, Energía y Turismo. 